\title{
Initiatives in Medical Volunteerism Aimed at Reducing the Burden of Orthopedic Disease in Low- and Middle- Income Countries
}

\author{
Albert Thomas Anastasio ${ }^{1 *}$ \\ ${ }^{1}$ School of Medicine, Emory University, Atlanta, USA \\ Corresponding Author: Albert Thomas Anastasio, School of Medicine, Emory University, 201 Dowman Dr, Atlanta, GA \\ 30322, USA. Tel: +1-2174189132, Email: albert.anastasio@emory.edu
}

Received December 1, 2018; Accepted May 15, 2019; Online Published May 29, 2019

\begin{abstract}
While infectious disease garners much attention and research funding globally, initiatives aimed at treating traumatic orthopedic injuries are underfunded despite such injuries accounting for a significant degree of morbidity worldwide. In recent years, a number of organizations have sought to alleviate this burden through treatment and educational initiatives in low- and middle-income countries (LMICs). Additionally, emerging developments in orthopedic technology are allowing for more expansive treatment options in resourcepoor environments. Distribution of both used and surplus orthopedic implants and components in LMICs and programs intended to encourage resident involvement with overseas volunteerism have proved beneficial in reducing the global burden of orthopedic disease. The goal of this manuscript is to educate readers about current initiatives in orthopedic volunteerism.

Keywords: Orthopedic, Volunteerism, Fracture Care, Medical Education
\end{abstract}

Citation: Anastasio AT. Initiatives in medical volunteerism aimed at reducing the burden of orthopedic disease in low- and middle-income countries Int J Travel Med Glob Health. 2019;7(2):45-47. doi:10.15171/ijtmgh.2019.10.

Introduction: The Staggering Burden of Orthopedic Disease in Low- and Middle-Income Countries Work to alleviate the burden of infectious disease has greatly reduced mortality rates from HIV/AIDS, malaria, and tuberculosis across the world and represents a tremendous success story in global health. ${ }^{1-8}$ What many health professionals may not realize, however, is that injuries account for roughly two times the number of deaths worldwide as these three diseases combined. Additionally, for each traumatic death recorded globally, 3 to 8 individuals remain permanently disabled, unable to provide for their families and without the capacity for upward economic mobility. ${ }^{9}$ Funding agencies are doing little to address this imbalance. For example, the Institute for Global Orthopedics and Traumatology (IGOT) (https://orthosurgery.ucsf.edu/outreach/programs/ igot_program.html) reports that for every $\$ 200$ spent on HIV research, $\$ 1$ is spent on research aimed at orthopedic injury. ${ }^{10}$

As the global economy continues to provide avenues for development in parts of the world that were once shut off from modern advances, mismatches arise between existing infrastructures and incoming technology. One such instance is an influx of vehicles, many of which are convenient for use in LMICs, but not well-equipped from a safety perspective (mopeds, motorcycles, open-backed trucks, etc). ${ }^{11}$ Such vehicles are brought into such countries well before a proper safety infrastructure is put in place, leading to a devastating number of road traffic-related incidents. Additionally, the lack of safety infrastructures at mass human gatherings can lead to stampede and subsequent orthopedic trauma. ${ }^{12}$ To illustrate this point, $95 \%$ of all operations at a hospital in Ghana were trauma-related procedures compared with $65 \%$ at a US hospital in San Francisco. ${ }^{13}$ Orthopedic disease is an underrecognized, underfunded, and shockingly significant contribution to the burden of disease in LMICs.

Much of the burden of orthopedic disabilty could be alleviated by urgent attention from healthcare professionals trained in orthopedic management. While roughly half of the world lacks access to primary care, 2/3 lack access to orthopedic care. ${ }^{9}$ To illustrate this shortage, there are 24 orthopedic surgeons in Ghana and 23,956 in the United States. ${ }^{13}$ Ghana has a population roughly $11 \mathrm{x}$ smaller than that of the US, yet the number of orthopedic surgeons in Ghana is almost 1000x smaller. Other studies have documented similar issues with access to orthopedic care in LMICs. ${ }^{14}$

Initiatives aimed at curbing the global burden of orthopedic disease can be subdivided into two categories: immediate

Copyright (C) 2019 The Author(s). This is an open-access article distributed under the terms of the Creative Commons Attribution License (http:// creativecommons.org/licenses/by/4.0), which permits unrestricted use, distribution, and reproduction in any medium, provided the original work is properly cited. 
and operative management of disease, and education of local healthcare personnel who can manage follow up and eventually participate in the care of the orthopedic patient. This article will focus on fracture management, as the need for such care in LMICs is great. This work will hopefully serve as a starting point for practitioners who would like to educate themselves and become involved in the management of orthopedic disease in LMICs.

\section{Treatment Initiatives: Immediate Stabilization, Operative Management, and Follow Up}

Thankfully, much is being done to mitigate the dearth of orthopedic specialty care in LMICs. Zirkle et al. published recently about the Surgical Implant Generation Network (SIGN) (https://signfracturecare.org/), a coalition aimed at providing equipment to orthopedic surgeons operating in underserved communities domestically and worldwide. ${ }^{15}$ Specifically, they have developed an intramedullary (IM) nail interlocking screw (SIGN) system which assists in the treatment of long-bone fractures. Unlike other IM nailing systems, the SIGN system does not require the use of C-arm imaging to achieve interlocking of the nail. C-arms and the personnel and training time needed for their operation represents a prohibitive barrier to many hospitals in LMICs who are interested in expanding their repertoire of orthopedic procedures. At the time of publication, the SIGN IM nail had been placed in 36000 patients in lower income countries with excellent success. ${ }^{15}$

A statement from an International Society for Prosthetics and Orthotics (ISPO) (https://www.ispoint.org/) -sponsored consensus conference recognized the need for the proper distribution of orthopedic materials, especially in war-torn regions where amputations are common. ${ }^{16}$ Although the ISPO's interest in expanded use of orthopedic technology adds potential bias, the obvious need for such materials should be recognized. As a broad summary, the ISPO statement calls for collaboration between local governments and non-profit disaster relief organizations in the provision of orthopedic technology. ${ }^{16}$

In the Unites States, a great deal of surplus orthopedic materials goes unused. The non-profit organization Orthopaedic Link (http://orthopaediclink.org/) is committed to mobilizing surplus orthopedic implants and instruments for use in LMICs. Another promising initiative is aimed at recycling expensive components for use in LMICs by resterilizing used orthopedic implants. ${ }^{17}$ Hardware should be reused with due caution against the transmission of infection (necessitating exquisite attention to detail in sterilization techniques), metal fatigue/implant failure, and the corrosive potential of implants leading to the release of toxic (ionic) compounds. Nonetheless, with adequate refurbishment, the re-use of implanted and subsequently removed plates and screws could greatly improve the capacity for fracture management in LMICs.

\section{Initiatives in Orthopedic Surgical Education}

To create a model of sustained orthopedic care in parts of the world currently lacking in such infrastructure, educational initiatives aimed at training local healthcare providers at managing disease constitute perhaps the most effective means for alleviating the global burden of orthopedic disease. Efforts to empower and mobilize a workforce created from a local population will provide long-term care that will continue well after the initial resources of a disaster-relief initiative are exhausted. ${ }^{18}$

Establishing a culture of education similar to what exists across medical and surgical residencies in the US would foster the development of orthopedic surgical training programs in LMICs. Instituting widespread morbidity and mortality ( $\mathrm{M} \& \mathrm{M})$ conferences, which are a mainstay of medical education in the US, would constitute a significant move in this direction. Sheth et al. took this one step further, calling for international M \& M conferences where a global forum would allow LMICs to share experiences and work with higher-income countries in a partnership to enhance patient care. ${ }^{19}$ Many organizations have been founded with orthopedic education as a primary goal. For example, the South Carolina Orthopaedic Association (https://www. scoanet.org/) has partnered with surgeons in Haiti at the Hopital de la Paix Orthopaedic Department to create the Haiti Global Orthopedic Resident Education Initiative. The aims of this project are to train Haitian surgeons to provide continuity of care for patients treated during volunteer projects and to expose Haitian surgeons to residency training methodology accredited by the Accreditation Council for Medical Graduate Education (ACGME).

Other organizations have been set up to provide orthopedic care directly. Operation Rainbow (http://operationrainbow. $\operatorname{org} /$ ) is, in their own words, a "nonprofit organization dedicated to performing free orthopedic surgery for indigent children and young adults in developing countries worldwide who do not have access to related medical procedures or equipment." In carrying out their mission, they have provided the equivalent of $\$ 80$ million USD worth of care in LIMCs at an expenditure of just US\$4.75 million. ${ }^{20}$ Similarly, Health Volunteers Overseas (HVOs) (https://hvousa.org/) is a non-profit organization committed to providing care in various medical fields. It has a wide range of ongoing projects for orthopedic surgeons in Bhutan, Bolivia, China, and many other locations, in which surgeons work together with local medical providers. Additionally, they have a fellowship opportunity for surgeons interested in working in LMICs who are committed to learning how to apply their skills in a resource-poor environment. HVOs also hosts an annual meeting at the American Academy of Orthopedic Surgeons along with the Société Internationale de Chirurgie Orthopédique et de Traumatologie (SICOT) (http://www. sicot.org/) and the World Orthopaedic Concern (WOC) (http://www.worldorthopaedicconcern.org/), two other organizations committed to the treatment of orthopedic disease in LMICs.

A few other organizations are providing both direct orthopedic care and education in LMICs. The creators of the SIGN IM nail, for example, have been active in surgical education in LMICs, training local providers on proper use of the nail and keeping tabs of the performance of the implant 
in a large database. ${ }^{15}$ This effort represents an emerging emphasis on empirical analysis of volunteerism in LMICs. Such initiatives will hopefully aid in determining the best practices to achieve maximal results in parts of the world where clinical research infrastructure has historically been lacking.

Perpetuation of the organizations listed above will occur only if orthopedic surgeons in-training are trained to value medical volunteerism. At the University of Washington, a survey indicated that $60 \%$ of orthopedic surgical residents would "be willing to commit to an international rotation." ${ }^{21}$ The desire to serve abroad seems to exist amongst residents, and residency programs are responding. An initiative out of the University of San Francisco, a renowned orthopedic surgery residency training facility, has encouraged their residents and faculty to pursue work globally in LMICs by providing funding and hosting service trips through the above mentioned IGOT. ${ }^{22}$ Residents can also volunteer through HVOs throughout their training as their schedule allows. These efforts will ensure the continuation of orthopedic volunteerism in LMICs.

\section{Conclusion}

A tremendous need exists for the timely provision of treatment for orthopedic disease in LMICs. Through the establishment and advancement of organizations committed to financial and material support, education, and volunteerism, the global burden of orthopedic disease can be mitigated.

\section{Conflict of Interest Disclosures}

The authors declare that they have no conflicts of interest.

\section{Ethical Approval}

Not applicable.

\section{Funding/Support}

None.

\section{References}

1. Kharsany AB, Karim QA. HIV infection and AIDS in sub-Saharan Africa: current status, challenges and opportunities. Open AIDS J. 2016;10:34-48. doi:10.2174/1874613601610010034.

2. Cohen GM. Access to diagnostics in support of HIV/AIDS and tuberculosis treatment in developing countries. Aids. 2007;21 Suppl 4:S81-87. doi:10.1097/01.aids.0000279710.47298.5c.

3. Bock P, Cox H. Acute care - an important component of the continuum of care for HIV and tuberculosis in developing countries. Anaesthesia. 2017;72(2):147-150. doi:10.1111/ anae. 13604.

4. Kumarasamy N, Venkatesh KK, Vignesh R, et al. Clinical outcomes among HIV/tuberculosis-coinfected patients developing immune reconstitution inflammatory syndrome after HAART initiation in South India. J Int Assoc Provid AIDS Care. 2013;12(1):28-31. doi:10.1177/1545109712457711

5. Vijayan T, Klausner JD. Integrating clinical services for HIV, tuberculosis, and cryptococcal disease in the developing world: a step forward with 2 novel diagnostic tests. J Int Assoc Provid AIDS Care. 2013;12(5):301-305. doi:10.1177/2325957413500472.

6. Charles M, Pape JW. Tuberculosis and HIV: Implications in the developing world. Curr HIV/AIDS Rep. 2006;3(3):139-144. doi:10.1007/bf02696658.

7. Tola HH, Tol A, Shojaeizadeh D, Garmaroudi G. Tuberculosis treatment non-adherence and lost to follow up among TB patients with or without HIV in developing countries: a systematic review. Iran J Public Health. 2015;44(1):1-11.

8. Gazzard B. Tuberculosis, HIV and the developing world. Clin Med (Lond). 2001;1(1):62-68. doi:10.7861/clinmedicine.1-1-62.

9. Dormans JP, Fisher RC, Pill SG. Orthopaedics in the developing world: present and future concerns. J Am Acad Orthop Surg. 2001;9(5):289-296. doi:10.5435/00124635-200109000-00002.

10. Institute for Global Orthopedics and Traumatology (IGOT). https:// orthosurgery.ucsf.edu/outreach/global/igot_program.html.

11. Gosselin RA, Spiegel DA, Coughlin R, Zirkle LG. Injuries: the neglected burden in developing countries. Bull World Health Organ. 2009;87(4):246-246a. doi:10.2471/BLT.08.052290.

12. Manoochehry S, Rasouli HR. Recurrent human tragedy during Hajj. Int J Travel Med Glob Health. 2017;5(1):36-37. doi:10.15171/ ijtmgh.2017.07.

13. Brouillette MA, Kaiser SP, Konadu P, Kumah-Ametepey RA, Aidoo AJ, Coughlin RC. Orthopedic surgery in the developing world: workforce and operative volumes in Ghana compared to those in the United States. World J Surg. 2014;38(4):849-857. doi:10.1007/ s00268-013-2314-0.

14. Gosselin RA, Gyamfi YA, Contini S. Challenges of meeting surgical needs in the developing world. World J Surg. 2011;35(2):258-261. doi:10.1007/s00268-010-0863-z.

15. Zirkle LG Jr. Injuries in developing countries--how can we help? The role of orthopaedic surgeons. Clin Orthop Relat Res. 2008;466(10):2443-2450. doi:10.1007/s11999-008-0387-0.

16. ISPO consensus conference on appropriate orthopaedic technology for low-income countries: conclusions and recommendations. International Society for Prosthetics and Orthotics. Prosthet Orthot Int. 2001;25(3):168-170. doi:10.1080/03093640108726598.

17. Magetsari R, Hilmy CR, van Horn JR, Busscher HJ, Verkerke GJ, van der Mei HC. Use and re-use of orthopedic implants in developing countries. J Biomed Mater Res B Appl Biomater. 2004;69(2):249250. doi:10.1002/jbm.b.30029.

18. Swidler A, Watkins SC. 'Teach a man to fish': The Doctrine of Sustainability and its effects on three strata of Malawian society. World Dev. 2009;37(7):1182-1196. doi:10.1016/j. worlddev.2008.11.002.

19. Sheth NP, Donegan DJ, Foran JR, Sugarman J. Global health and orthopaedic surgery-A call for international morbidity and mortality conferences. Int J Surg Case Rep. 2015;6c:63-67. doi:10.1016/j.ijscr.2014.11.074.

20. Operation Rainbow. http://operationrainbow.org/.

21. Jense RJ, Howe CR, Bransford RJ, Wagner TA, Dunbar PJ. University of Washington orthopedic resident experience and interest in developing an international humanitarian rotation. Am J Orthop (Belle Mead NJ). 2009;38(1):E18-20.

22. Rovinsky D, Brown HP, Coughlin RR, Paiement GD, Bradford DS Overseas volunteerism in orthopaedic education. J Bone Joint Surg Am. 2000;82(3):433-436. doi:10.2106/00004623-20000300000015. 